ability to switch devices on and off, send reminders, chat to other young people and to track their own data. Some caution was expressed about a device monitoring sleep (or other behaviours that parents may want to know about). It was important for participants to know where the data will be stored, who had access to it and what the information will be used for.

Conclusion: Young people have clear opinions as to what they like and don't like with respect to wearable data collection devices. As well as personal preferences, age specific considerations were highlighted by the young people. It is therefore imperative not only to involve young people as research participants but also to involve them at early stages of research including trial design to ensure acceptability of data collection methods including the design of any devices proposed.

Acknowledgement: Your Rheum is the national youth advisory panel of the Barbara Ansell National Network for Adolescent Rheumatology BANNAR. We would like to thank all of the young people who took part and other individuals who facilitated their involvement including BANNAR members and Versus Arthritis Young People Coordinators.

Disclosure of Interests: Laura Lunt Grant/research support from: Your Rheum is funded by the Arthritis Research UK grant 20164, via the Centre for Adolescent Rheumatology at UCL. The event described here was funded by Eli Lilly., Stephanie Shoop-Worrall Grant/research support from: Your Rheum is funded by the Arthritis Research UK grant 20164, via the Centre for Adolescent Rheumatology at UCL. The event described here was funded by Eli Lilly., Sonia Khanom Grant/ research support from: Your Rheum is funded by the Arthritis Research UK grant 20164, via the Centre for Adolescent Rheumatology at UCL. The event described here was funded by Eli Lilly., Imogen Bolger: None declared, Antoniu L Fantana Shareholder of: Eli Lilly and Company, Employee of: Eli Lilly and Company, Mary A Short Shareholder of: Eli Lilly and Company, Employee of: Eli Lilly and Company, janet McDonagh Grant/research support from: Your Rheum is funded by the Arthritis Research UK grant 20164, via the Centre for Adolescent Rheumatology at UCL. The event described here was funded by Eli Lilly., Consultant for: The event described here was funded by Eli Lilly.

DOI: 10.1136/annrheumdis-2019-eular.1923

\section{OP0289-PARE THE ROLE OF PATIENTORGANISATIONS ON THE EDUCATION AND ESTABLISHMENT OF RHEUMATOLOGY NURSE}

Androulla Phoka Charalambous. Cyprus League Against Rheumatism, Nicosia, Cyprus

Background: Rheumatology Nurse is a practitioner well educated or with a specialty on Rheumatology diseases who help people with RMD's to live as fully independently as possible. A Big Part of the rheumatology nurse's job is to help patients with rheumatic diseases to release their pain and manage their symptoms. In Europe, the use of rheumatology nurses for the treatment of rheumatism is becoming more and more popular. In my country, this is not the case. There is still a lot of work to be done to apply the rheumatology practitioner. That's why our organization is pushing the government to implement in the system Rheumatology Nurse.

Objectives: During discussions between our organization and the Ministry of Health for asking the use of rheumatology nurse in the rheumatology outpatient clinic, since we don't have a rheumatology inpatient clinic, Ministry refused to give the authorisation with the justification of not having any nurse specialist, educated or interested to be educated on the rheumatology fields. So this was a challenge for our organization: The "Nurse Education on Rheumatology".

Methods: The aim had various steps to achieve. The first step was to inform Government Nursing Services the significant of Rheumatology Nurse. The second step was to discuss with them the need for educated Nurses on rheumatology and their opinion. Third to persuade them to educate nurses on Rheumatology by organizing an Education Program. And the Last one but Very Important, part of the Education Program to be our organization not only as a presenter but to have an actual role as an associate with opinion, on the structure and themes of the program.

Results: The organisation had many meetings with the Government Nursing Services. The officers become very excited about the idea of having a New Education Program on such an interesting theme. After having a number of meetings about the development of the Program and after escaping the barriers, the program was done: Educational Program title "Patient Care with Rheumatic Diseases". Duration of the training: 3 months, 1 day per week, with 3 days of Clinical Exercise on outpatient Rheumatology Clinic and 1 Day on the Care Departmentpreparation and delivery of biological and biosimilar factors. Success in the Program requires Final Written Evaluation (60\%) and Case study (Presentation). Patient Organization: 5 Lectures- Workshops. The education took place from October to December 2018. 27 Nurses have been educated. The program includes between a lot of Lectures, Patient Raise Awareness and Patient Perspective which were given by our Patient Organization. The lectures were given from Organizations'Psychologist staff and from Volunteers Patient Experts for giving the patient perception for the treatment and for the self-management of their condition and Organization's Big Role regarding the disease awareness, treatment, and management. Organization conducted a survey among the participants and the results are very encouragingly to continue the tries to implement the Rheumatology Nurse in the outpatient and inpatient Rheumatology Clinics of the Country. At the question "after training will you be interested in working as a Rheumatology Nurse in a Rheumatology Clinic?'The answer was YES for the $100 \%$ of the participants.

Conclusion: The importance of nurses' involvement in the treatment of rheumatic diseases is enormous. This has been demonstrated in Europe. Our Organization shows the significant role of nurses for achieve the maximum results on the patient treatment. Also the organization has been able to prove the importance of the contribution of patients to better treatment of the disease. It has also demonstrated to the state the need for a Rheumatology Nurse and refutes their excuses. Disclosure of Interests: None declared

DOI: 10.1136/annrheumdis-2019-eular.1105

FRIDAY, 14 JUNE 2019

\section{Rheumatoid arthritis - etiology, pathogenesis and animal models}

\section{OP0290 HOTAIR IS INVOLVED IN JOINT PATTERNING BY REGULATING FGFR2, BMP2 AND WNT PATHWAYS}

Muriel Elhai, Mojca Frank-Bertoncelj, Kerstin Klein, Oliver Distler, Caroline Ospelt. Center of Experimental rheumatology, Department of Rheumatology, University Hospital Zurich, Zürich, Switzerland

Background: Different forms of chronic arthritis such as rheumatoid arthritis (RA) or spondyloarthritis show a typical pattern of joint involvement. However, mechanisms involved in this patterning remain unknown. Synovial fibroblasts (SF) isolated from different joint regions, differ in their epigenetic landscape, their gene expression and their function. The long non-coding RNA HOTAIR, which is an important regulator of the epigenetic landscape, was found to be exclusively expressed in joints of the lower extremity.

Objectives: We aim to clarify the role of HOTAIR in SF.

Methods: HOTAIR was silenced in knee SF isolated from patients with osteoarthritis (OA) by HOTAIR GapmeR using Lipofectamine, and changes in gene expression were measured by next generation RNA sequencing (Illumina NovaSeq 6000$)$ after $48 \mathrm{~h}(\mathrm{n}=3)$. Pathway analysis was performed using STRING protein network, and regulated genes were confirmed by real-time PCR in SF silenced for HOTAIR from OA and RA-patients $(n=8)$ and compared with control SF transfected with Antisense LNA GapmeR negative control. The time course of targeted genes was studied with and without cycloheximide (10ug/ml $6 \mathrm{~h}$ and $24 \mathrm{~h})$ $(n=2)$. To assess the effect of HOTAIR silencing on the canonical Wnt pathway, we employed the TOP/FOP reporter system using the dual-luciferase kit $(n=3)$. Regulation of HOTAIR and targeted genes expression was studied after stimulation with TNF- $\alpha(n=17)$, IL1, LPS, TLR2, TLR4 and poly I:C $(n=5)$. The expression of HOTAIR and TNF- $\alpha$ were measured in synovial tissue from RA-patients $(n=7)$ by qPCR.

Results: STRING analysis identified among others three significantly enriched pathways, namely FGF, BMP and Wnt, including 19 genes differentially expressed between SF silenced for HOTAIR and controls according to p-value $(<0.05)$ and log ratio (>2.5 or <-2.5). Real-time PCR confirmed 6 genes: FGFR2 (5 \pm 0.1 -fold downregulated; $p<0.01$ ), FGF7 (1.9 \pm 0.4 -fold downregulated; $p=0.02$ ) BMP2 (1.7 \pm 0.5-fold upregulated; $p<0.01)$, LGR5 (3.4 \pm 0.3 -fold downregulated $\mathrm{p}=0.02)$, CTNNB1 (beta-catenin) $(1.5 \pm 0.5$-fold upregulated; $p<0.01)$ and GSK3 $\beta$ (1.8 \pm 0.2 -fold downregulated; $p=0.03)$. There was a trend for LRP6 $(p=0.06)$. HOTAIR expression and FGFR2 and FGF7 expression were correlated $(r=0.67$ and $0.55 ; p<0.01)$ as well as HOTAIR and BMP2 $(r=-0.45 ; p<0.05)$. Time course of gene expression peaked after 30-48 hours. Adjunction of cycloheximide, blocking translation, did not change gene expression of BMP2 and CTNNB1 in SF silenced for HOTAIR, suggesting a direct effect of HOTAIR on these genes. FGFR2, FGF7, LGR5, LRP6 and GSK3 3 expression were normalized with cycloheximidine indicating the need of a protein to mediate HOTAIR effect on FGF pathway and on Wnt pathway. TopFlash assay confirmed the role of HOTAIR in regulation of Wnt pathway by $1.7 \pm 0.2$-fold decrease in Wnt activation after HOTAIR silencing $(p=0.02)$. Stimulation by TNF- $\alpha$ led to a $2.1 \pm 0.3$ decrease in HOTAIR expression $(p<0.0001)$. Following TNF- $\alpha$ stimulation, FGFR2 and LGR5 expression decreased ( $p=0.0001$ and 0.0004 , respectively), whereas there was an increase in BMP2 expression $(p<0.0001)$, consistent with the effect of silencing HOTAIR on these genes. There was a decrease in HOTAIR expression following LPS $(p=0.006)$, and poly I:C ( $p=0.003)$. HOTAIR and TNF- $\alpha$ were inversely correlated in the synovium of RA patients $(r=-0.79 ; p<0.05)$.

Conclusion: Our study highlights three main pathways regulated by HOTAIR and involved in joint patterning: FGFR2/FGF7, BMP2 and LRP6/LGR5/GSK3ß/ 
beta-catenin (Wnt). These pathways are known to play a role in normal joint development, but are also involved in inflammation, proliferation and apoptosis. Consistently, we observed that HOTAIR and targeted genes were selectively downregulated by TNF- $\alpha$ suggesting a role of HOTAIR in the joint specific regulation of TNF- $\alpha$ mediated inflammatory arthritis.

Disclosure of Interests: Muriel ELHAI: None declared, Mojca Frank-Bertoncelj None declared, Kerstin Klein: None declared, Oliver Distler Grant/research sup port from: Prof. Distler received research funding from Actelion, Bayer, Boehringe Ingelheim and Mitsubishi Tanabe to investigate potential treatments of scleroderma and its complications, Consultant for: Prof. Distler has/had consultancy relationship within the last 3 years with Actelion, AnaMar, Bayer, Boehringer Ingelheim, ChemomAb, espeRare foundation, Genentech/Roche, GSK, Inventiva, Italfarmaco, iQvia, Lilly, medac, Medlmmune, Mitsubishi Tanabe Pharma, Pharmacyclics, Novartis, Pfizer, Sanofi, Serodapharm and UCB in the area of potential treatments of scleroderma and its complications. In addition, he had/has consultancy relationship within the last 3 years with A. Menarini, Amgen, Abbvie, GSK, Mepha, MSD, Pfizer and UCB in the field of arthritides and related disorders, Caroline Ospelt: None declared

DOI: 10.1136/annrheumdis-2019-eular.4370

\section{OP0291 ACTIVATION OF THE DEACETYLASE SIRTUIN-1 COUNTERACTS THE ACTIVATED AND PROANGIOGENIC PROFILE OF ENDOTHELIAL CELLS IN RHEUMATOID ARTHRITIS AND ALLEVIATES EXPERIMENTAL ARTHRITIS}

Agathe Leblond ${ }^{1}$, Sonia Pezet ${ }^{1}$, Anne Cauvet ${ }^{1}$, Claudine Casas ${ }^{1}$, Julie Pires Da Silva ${ }^{2}$, Roxane Hervé ${ }^{3}$, Luca Semerano ${ }^{3}$, Christophe Lemaire ${ }^{2}$, Yannick Allanore ${ }^{1,4}$, Jérôme Avouac ${ }^{1,4}$. ${ }^{1}$ Paris Descartes University, INSERM U1016, Paris, France; ${ }^{2}$ Université Versailles St-Quentin, UMR-S 1180, ChâtenayMalabry, France; ${ }^{3}$ Paris 13 University, Inserm UMR 1125, Bobigny, France; ${ }^{4}$ Paris Descartes University, Cochin Hospital, Rheumatology, Paris, France

Background: Angiogenesis through the recruitment and activation of endothelial cells (ECs) is a crucial event to promote the development of the hyperplasic proliferative synovium in rheumatoid arthritis (RA).

Objectives: Our aim was to decipher the phenotype of ECs derived from circulating progenitors issued from patients with $R A$.

Methods: Proliferation capacities between RA and control ECs was compared using the $x$ CELLigence ${ }^{\text {TM }}$ RTCA System. rh-TNF $\alpha$-induced EC activation was analyzed by adhesion cell expression, VEGF synthesis and stress fiber formation. Angiogenic properties of ECs were assessed in vitro by tube formation on Matrigel and migration capacities through VEGF stimulation in modified Boyden chambers, and in vivo in a mouse model of tumoral neovascularization. Microarray experiments were then performed on Affymetrix GeneChip ${ }^{\circledR}$ Human Exon 1.0 ST Arrays in ECs issued from 18 RA patients compared to 11 age and sex-matched healthy controls to identify gene candidates relevant to pathological angiogenesis. Expression of identified candidates was assessed by RT-PCR and western blots in ECs and by immunohistochemistry in the synovial tissue of RA patients and controls. Their functional importance was then evaluated in vitro after gene invalidation by siRNA and gene overexpression via adenovirus, and in vivo in the mouse model of methyl-BSA-induced arthritis.

Results: EPC-derived ECs issued from RA patients displayed higher proliferation rate, greater sensitization to TNF $\alpha$, with increased VEGF production, ICAM/ VCAM expression, and more prominent stress fiber formation, as well as enhanced angiogenic capacities, characterized by accelerated tube formation and increased migration capacities through VEGF stimulation, compared co control ECs. The subcutaneous transplantation of murine colon carcinoma (CT-26) cells with RA ECs in CB17-SCID mice markedly amplified tumor growth and intratumoral neovessel density, compared to the transplantation of control ECs.

Supervised microarray analyses identified the $\mathrm{NAD}^{+}$-dependent protein deacetylase sirtuin-1 (SIRT1) as a relevant gene candidate. A strikingly decreased SIRT1 gene/protein expression and enzyme activity was detected in RA ECs. A markedly decreased SIRT1 expression was also observed in RA synovium, and more particularly in blood vessels.

Invalidation of SIRT1 with specific siRNA in control ECs was associated with a proliferative and activated profile upon TNF $\alpha$ stimulation, trough the acetylation of p53 and p65, and with the proangiogenic capacities through the upregulation of the matricellular protein CYR61. Conditional deletion of SIRT1 in ECs trough a Cre-LoxP recombination system increased angiogenesis and worsened signs of arthritis and in experimental models of tumor angiogenesis and methyl-BSAinduced arthritis. Conversely, overexpression of SIRT1 via adenovirus in RA ECs reversed this activated and proangiogenic phenotype, and activation of SIRT1 with resveratrol alleviated signs of experimental methyl-BSA-induced arthritis. Conclusion: SIRT1 expression is reduced in synovial blood vessels of patients with RA. SIRT1 invalidation in control ECs reproduces the proliferative, activated and proangiogenic profile of RA ECs, and these effects were reversed by SIRT1 overexpression in RA ECs. These results support the implication of SIRT1 in RA synovial neoangiogenesis and may have direct therapeutic implications, since targeting angiogenesis, and especially SIRT1, might be used as a complementary therapeutic approach in RA.

Acknowledgement: This work was supported by the French Society of Rheumatology (SFR), Arthritis R\&D and a research grand from Pfizer ("Bourse Passerelle").

Disclosure of Interests: Agathe Leblond: None declared, Sonia Pezet: None declared, Anne Cauvet: None declared, Claudine Casas: None declared, Julie Pires Da Silva: None declared, Roxane Hervé: None declared, Luca Semerano Grant/research support from: pfizer, Speakers bureau: pfizer, roche, msd, bms, Christophe Lemaire: None declared, Yannick Allanore Grant/research support from: Inventiva, F Hoffman La-Roche, Sanofi, BMS, Pfizer, Consultant for: Actelion, Bayer, BMS, Boehringer, Roche, Sanofi, Jérôme Avouac Grant/research support from: research grant from Pfizer

DOI: 10.1136/annrheumdis-2019-eular.4516

\section{\begin{tabular}{|l|l}
\hline OP0292 THE HELPFUL EIGHT: KEY SPLICING MACHINERY \\
\hline
\end{tabular} ELEMENTS IN LEUKOCYTE SUBSETS MAY IMPROVE THE TYPIFICATION OF THE DISEASE IN RHEUMATOID ARTHRITIS PATIENTS}

Alejandro Ibañez-Costa ${ }^{1}$, Carlos Perez-Sanchez ${ }^{1}$, Nuria Barbarroja Puerto ${ }^{1}$, Mercedes del Río-Moreno ${ }^{1,2}$, Sergio Pedraza-Arévalo ${ }^{1,2}$, Alejandra M. PatiñoTrives $^{1}$, María Luque-Tévar ${ }^{1}$, Maria del Carmen Abalos-Aguilera ${ }^{1}$, Pedro Seguí Azpilcueta ${ }^{1}$, Pérez Sánchez Laura ${ }^{1}$, Jerusalem Calvo Gutierrez ${ }^{1}$, Rafaela Ortega Castro ${ }^{1}$, Alejandro Escudero Contreras ${ }^{1}$, Eduardo Collantes Estevez ${ }^{1}$,

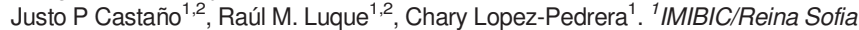
Hospital/University of Cordoba, Cordoba, Spain; ${ }^{2}$ Department of Cell Biology, Physiology and Immunology, University of Cordoba, Cordoba, Spain

Background: The Splicing, a process of removing introns of RNA to produce mature messenger RNA, is exerted by an intricate macromolecular complex, the spliceosome. The alternative splicing, key mechanism that provides transcription and protein diversity, is dysregulated in various genetic, metabolic and inflammatory diseases. Splicing variants could contribute to the development and severity of pathologies of crucial importance.

Objectives: The aim of this study was the identification and characterization of alterations in the splicing machinery of leukocyte subsets of Rheumatoid Arthritis (RA) patients, as well as their influence on the activity of the disease and its atherothrombotic profile.

Methods: Monocytes, lymphocytes and neutrophils from 74 RA patients and 29 healthy donors (HD) were purified, and 45 elements of the splicing machinery were evaluated using a microfluidic qPCR array (Fluidigm). In parallel, the clinical status of the patients and the carotid intima-media thickness (CIMT) were assessed, along with an extensive serological evaluation, including autoantibod ies, oxidative stress markers, inflammatory molecules and prothrombotic mediators. Correlation and association studies and logistic models among those clinica and analytical parameters were performed. Finally, leukocytes from HD were incubated with purified ACPAs-IgG from RA patients and changes promoted in both, splicing machinery and leukocyte inflammatory profile, were assessed.

Results: A significantly altered expression of spliceosome components was found in all the leukocyte subsets: 21,17 and 27 components were differentially expressed in monocytes, lymphocytes and neutrophils, respectively, in RA patient's vs HD. The levels of those altered components were associated to ACPA positivity, high disease activity (DAS28>5.1), early atherosclerosis and radiological involvement. Correlations with inflammatory mediators, oxidative stress markers and NETosis were further identified. Interestingly, eight components, belonging to the major spliceosome (SNRNP70, SNRNP200 and U2AF2), minor spliceosome (RNU4ATAC), and splicing factors (RBM3, RBM17, KHDRS1 and SRSF10) were found simultaneously altered in the three leukocyte subtypes. Logistic regression models, built with levels of simultaneously altered spliceosome components, generated several signatures related to RA disease features that were able to: i) discriminate between RA patients and HD; ii) classify patients with high disease activity (DAS28 $>5.1$ ); iii) recognize patients with radiological involvement; and iv) identify patients showing atheroma plaques. In vitro studies showed that the purified ACPAs of RA patients modulated the expression of these components.

Conclusion: 1) The splicing machinery is profoundly altered in leukocytes from RA patients and closely related to the activity of the disease, including inflammation, autoimmunity and articular involvement, as well as with the occurrence of early atherosclerosis. 2) Evaluation of the common altered components of the spliceosome in leukocytes subsets might be used to typify the disease, avoiding complementary ultrasound or radiological tests.

Acknowledgement: MINECO (FJCl-2016-30825) and ISCIII (PI18/00837 and RIER RD16/0012/0015) co-funded with FEDER.

Disclosure of Interests: None declared

DOI: 10.1136/annrheumdis-2019-eular.7066 